\title{
Structural insights into plasticity and discovery of remdesivir metabolite GS-441524 binding in SARS-CoV-2 macrodomain
}

Xiaomin $\mathrm{Ni}^{1,2 \#}$, Martin Schröder ${ }^{1,2 \#}$, Vincent Olieric ${ }^{3}$, May E. Sharpe ${ }^{3}$, Victor HernandezOlmos $^{4}$, Ewgenij Proschak ${ }^{2,4}$, Daniel Merk ${ }^{2}$, Stefan Knapp ${ }^{1,2^{*}}$, Apirat Chaikuad ${ }^{1,2^{*}}$

${ }^{1}$ Structural Genomics Consortium, Buchmann Institute for Molecular Life Sciences, 60438 Frankfurt am Main, Germany

${ }^{2}$ Institute of Pharmaceutical Chemistry, Goethe University Frankfurt, 60438 Frankfurt am Main, Germany

${ }^{3}$ Swiss Light Source, Paul Scherrer Institute, 5232 Villigen, Switzerland.

${ }^{4}$ Fraunhofer Institute for Translational Medicine and Pharmacology ITMP, Theodor-Stern-Kai 7, 60596 Frankfurt, Germany

\# These authors contributed equally.

* Correspondence: Stefan Knapp: knapp@ @ pharmchem.uni-frankfurt.de; Apirat Chaikuad:

chaikuad@pharmchem.uni-frankfurt.de

Supplementary information

\begin{tabular}{|l|l|}
\hline $\begin{array}{l}\text { Supplementary figure s1. }\left|F_{\mathrm{O}}\right|-\left|F_{\mathrm{C}}\right| \text { omitted electron density map contoured at } 3 \sigma \\
\text { for the bound ligands. }\end{array}$ & $\mathrm{S} 2$ \\
\hline Supplementary table s1. Details of recombinant SARS-CoV-2 macrodomain. \\
\hline Supplementary Table s2. Data collection and refinement statistics. & $\mathrm{S} 3$ \\
\hline Supplementary method. Synthesis of GS-441524 monophosphate & $\mathrm{S} 4$ \\
\hline
\end{tabular}


HEPES

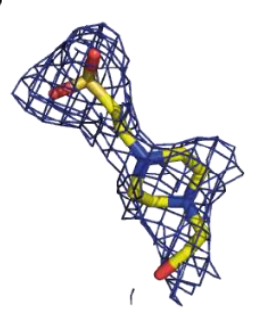

MES

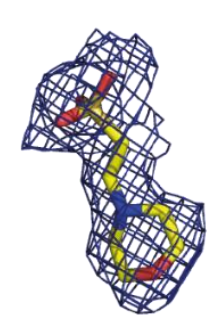

adenosine

conformation 2

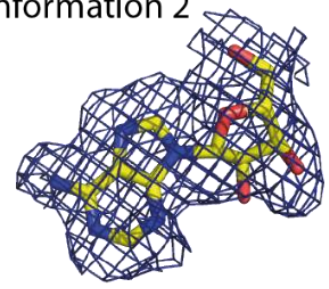

ADP-ribose

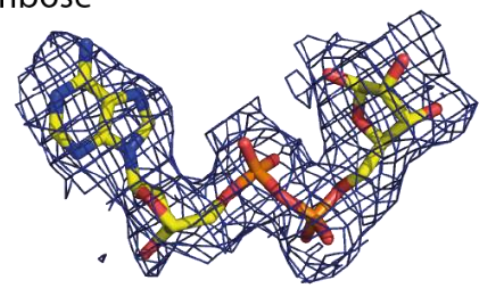

GMP

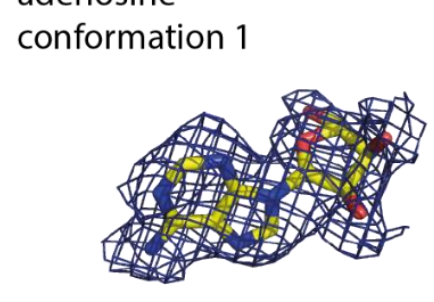

adenosine

ADP-ribose-2-phosphate

(ADPRP)

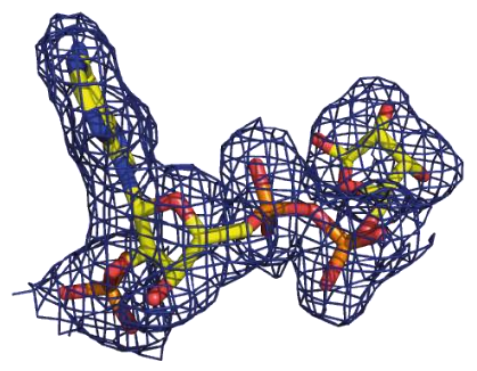

GS-441524

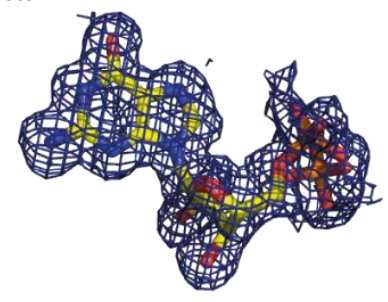

Supplementary Figure s1. $\left|F_{\mathrm{O}}\right|-\left|F_{\mathrm{C}}\right|$ omitted electron density map contoured at $3 \sigma$ for the bound ligands. 
Supplementary table s1. Details of recombinant SARS-CoV-2 macrodomain.

\begin{tabular}{|l|c|l|}
\hline & Vector & \multicolumn{1}{c|}{ Recombinant protein sequence } \\
\hline $\begin{array}{l}\text { SAR-CoV-2 } \\
\text { macrodomain }\end{array}$ & pET-28a(+) & $\begin{array}{l}\text { MGSSHHHHHHSSGENLYFQGHMVNSFSGYLLTDNVYIKNADIVEEAK } \\
\text { KVKPTVVVNAANVYLKHGGGVAGALNKATNNAMQVESDDYIATNGP } \\
\end{array}$ \\
& & $\begin{array}{l}\text { LKVGGSCVLSGHNLAKHCLHVVGPNVNKGEDIQLLSSAYENFNQHEVLL } \\
\text { APLLSAGIFGADPIHSLRVCVDTVRTNVYLAVFDKNLYDKLVSSFLEMK }\end{array}$ \\
\hline
\end{tabular}


Supplementary Table s2. Data collection and refinement statistics.

\begin{tabular}{|c|c|c|c|}
\hline Complex & apo/HEPES & apo/MES & ADP-ribose \\
\hline PDB codes & 6ywk & 6ywm & 6ywl \\
\hline Beamline & SLS X06SA & SLS X06SA & SLS X06SA \\
\hline \multicolumn{4}{|l|}{ Data Collection } \\
\hline Resolutiona $(\AA)$ & $49.09-2.20(2.28-2.20)$ & $49.22-2.16(2.24-2.16)$ & $48.83-2.50(2.64-2.50)$ \\
\hline Space group & $P 212121$ & $P 212121$ & $P 212121$ \\
\hline Cell dimensions & $\begin{array}{c}\mathrm{a}=39.2, \mathrm{~b}=111.8, \mathrm{c}=196.4 \AA \\
\alpha=\beta=\gamma=90.0\end{array}$ & $\begin{array}{c}\mathrm{a}=37.8, \mathrm{~b}=109.1, \mathrm{c}=114.4 \AA \\
\alpha=\beta=\gamma=90.0\end{array}$ & $\begin{array}{c}\mathrm{a}=38.4, \mathrm{~b}=111.9, \mathrm{c}=195.3 \AA \\
\alpha=\beta=\gamma=90.0^{\circ}\end{array}$ \\
\hline $\begin{array}{l}\text { Number of unique } \\
\text { reflections }^{\mathrm{a}}\end{array}$ & $45,087(4,348)$ & $26,281(2,558)$ & $30,002(4,288)$ \\
\hline Completeness ${ }^{\mathrm{a}}(\%)$ & $100.0(99.9)$ & $100.0(100.0)$ & $99.4(99.3)$ \\
\hline $\mathrm{I} / \sigma \mathrm{I}^{\mathrm{a}}$ & $10.7(2.0)$ & $8.3(2.0)$ & $6.9(1.9)$ \\
\hline $\operatorname{Rmerge}^{a}(\%)$ & $0.138(0.925)$ & $0.162(0.873)$ & $0.199(0.930)$ \\
\hline$C C(1 / 2)^{a}$ & $0.998(0.762)$ & $0.995(0.787)$ & $0.990(0.736)$ \\
\hline Redundancy ${ }^{\mathrm{a}}$ & $8.5(7.9)$ & $6.7(6.9)$ & $5.6(5.9)$ \\
\hline \multicolumn{4}{|l|}{ Refinement } \\
\hline $\begin{array}{l}\text { Number atoms in } \\
\text { refinement }(\mathrm{P} / \mathrm{L} / \mathrm{O})^{\mathrm{b}}\end{array}$ & $6,496 / 15 / 424$ & $3,885 / 24 / 287$ & $6,472 / 180 / 248$ \\
\hline $\mathrm{B}$ factor $(\mathrm{P} / \mathrm{L} / \mathrm{O})^{\mathrm{b}}\left(\AA^{2}\right)$ & $39 / 76 / 48$ & $34 / 57 / 39$ & $40 / 31 / 38$ \\
\hline$R_{\text {fact }}(\%)$ & 17.6 & 17.5 & 18.9 \\
\hline$R_{\text {free }}(\%)$ & 21.4 & 22.9 & 22.3 \\
\hline rmsd bond $(\AA)$ & 0.013 & 0.013 & 0.010 \\
\hline rmsd angle $\left(^{\circ}\right)$ & 1.4 & 1.3 & 1.1 \\
\hline \multicolumn{4}{|l|}{ Molprobity Ramachandran } \\
\hline Favor (\%) & 99.65 & 99.01 & 98.11 \\
\hline Outlier (\%) & 0 & 0 & 0 \\
\hline Crystallization condition & $\begin{array}{c}\text { 33\% broad-molecular- } \\
\text { weight PEG smears, } 0.1 \\
\mathrm{M} \mathrm{MgCl}_{2}, 0.1 \text { M HEPES, } \\
\text { pH } 7.0\end{array}$ & $\begin{array}{c}\text { 23\% PEG 6000, } 0.1 \mathrm{M} \\
\mathrm{MgCl}_{2}, 5 \% \text { ethylene } \\
\text { glycol, 0.1 M MES, pH } \\
6.0 \\
\end{array}$ & $\begin{array}{c}27 \% \text { PEG 4000, } 0.2 \mathrm{M} \\
\text { sodium acetate, } 0.05 \mathrm{M} \\
\mathrm{MgCl}_{2}, 0.1 \mathrm{M} \text { tris, } \mathrm{pH} 8.0\end{array}$ \\
\hline
\end{tabular}

a Value in brackets indicates high-resolution shell statistics.

$\mathrm{b} \mathrm{P} / \mathrm{L} / \mathrm{O}$ indicates protein, ligands and others.

${ }^{c}$ rmsd indicates root-mean-square deviation. 
Supplementary Table s2. (continued) Data collection and refinement statistics.

\begin{tabular}{|c|c|c|c|c|}
\hline Complex & Adenosine & GMP & ADPRP & GS-441524 \\
\hline PDB codes & $7 \mathrm{bf3}$ & $7 \mathrm{bf} 4$ & $7 \mathrm{bf5}$ & $7 \mathrm{bf6}$ \\
\hline Beamline & SLS X06SA & SLS X06DA & SLS X06SA & SLS X06SA \\
\hline \multicolumn{5}{|l|}{ Data Collection } \\
\hline Recolutiona ( & $48.99-2.00$ & $36.26-1.55$ & $48.80-2.05$ & $48.43-2.15$ \\
\hline Resolution (A) & $(2.07-2.00)$ & $(1.60-1.55)$ & $(2.12-2.05)$ & $(2.23-2.15)$ \\
\hline Space group & $P 212121$ & $P 41$ & $P 212121$ & $C 2$ \\
\hline \multirow[t]{2}{*}{ Cell dimensions } & $\begin{array}{c}a=39.2, b=111.4, \\
c=196.0 \AA\end{array}$ & $\mathrm{a}=\mathrm{b}=72.5, \mathrm{c}=33.4 \AA$ & $\begin{array}{c}a=38.6, b=111.3, \\
c=195,2 \AA\end{array}$ & $\begin{array}{c}\mathrm{a}=157.2, \mathrm{~b}=30.5 \\
\mathrm{c}=111,7 \AA\end{array}$ \\
\hline & $\alpha=\beta=\gamma=90.0$ & $\alpha=\beta=\gamma=90.0$ & $\alpha=\beta=\gamma=90.0^{\circ}$ & $\alpha=\gamma=90.0^{\circ}, \beta=119.9^{\circ}$ \\
\hline $\begin{array}{l}\text { Number of unique } \\
\text { reflections }^{\mathrm{a}}\end{array}$ & $59,412(5,774)$ & $25,315(2,287)$ & $53,878(5,196)$ & $25,440(2,459)$ \\
\hline Completeness ${ }^{\mathrm{a}}(\%)$ & $100.0(100.0)$ & $99.2(92.8)$ & $99.7(99.8)$ & $99.1(99.2)$ \\
\hline $\mathrm{I} / \sigma \mathrm{I}^{\mathrm{a}}$ & $10.9(2.0)$ & $13.8(2.6)$ & $8.3(1.9)$ & $10.7(1.9)$ \\
\hline Rmerge $^{a}(\%)$ & $0.127(0.885)$ & $0.064(0.349)$ & $0.141(0.839)$ & $0.090(0.755)$ \\
\hline $\mathrm{CC}(1 / 2)^{\mathrm{a}}$ & $0.998(0.735)$ & $0.998(0.807)$ & $0.995(0.695)$ & $0.998(0.677)$ \\
\hline Redundancy ${ }^{a}$ & $7.5(7.5)$ & $6.1(3.3)$ & $6.2(6.3)$ & $5.3(5.2)$ \\
\hline \multicolumn{5}{|l|}{ Refinement } \\
\hline $\begin{array}{l}\text { Number atoms in } \\
\text { refinement }(\mathrm{P} / \mathrm{L} / \mathrm{O})^{\mathrm{b}}\end{array}$ & $6,538 / 38 / 628$ & $1,329 / 48 / 241$ & $6,500 / 160 / 569$ & $3,838 / 63 / 168$ \\
\hline $\begin{array}{c}\text { B factor }(\mathrm{P} / \mathrm{L} / \mathrm{O})^{\mathrm{b}} \\
\left(\AA^{2}\right)\end{array}$ & $29 / 60 / 37$ & $14 / 13 / 30$ & $28 / 41 / 35$ & $49 / 39 / 43$ \\
\hline$R_{\text {fact }}(\%)$ & 17.5 & 13.8 & 17.7 & 18.0 \\
\hline$R_{\text {free }}(\%)$ & 21.7 & 17.4 & 21.7 & 22.6 \\
\hline $\operatorname{rmsd~bond~}^{c}(\AA)$ & 0.013 & 0.018 & 0.014 & 0.012 \\
\hline rmsd angle $\left(^{\circ}\right)$ & 1.4 & 1.7 & 1.4 & 1.4 \\
\hline \multicolumn{5}{|l|}{ Molprobity } \\
\hline \multicolumn{5}{|l|}{ Ramachandran } \\
\hline Favor (\%) & 98.94 & 99.40 & 97.64 & 99.00 \\
\hline Outlier (\%) & 0 & 0 & 0 & 0 \\
\hline $\begin{array}{l}\text { Crystallization } \\
\text { condition }\end{array}$ & $\begin{array}{c}33 \% \text { broad- } \\
\text { molecular-weight } \\
\text { PEG smears, } 0.1 \mathrm{M} \\
\mathrm{MgCl}_{2}, 0.1 \mathrm{M} \text { tris, } \\
\text { pH } 7.0\end{array}$ & $\begin{array}{c}30 \% \text { PEG } 4000,0.2 \\
\mathrm{M} \text { sodium acetate, } \\
0.1 \mathrm{MgCl}_{2}, 0.1 \mathrm{M} \\
\text { tris, } \mathrm{pH} 8.3\end{array}$ & $\begin{array}{c}30 \% \text { broad- } \\
\text { molecular-weight } \\
\text { PEG smears, } 0.1 \mathrm{M} \\
\mathrm{MgCl}_{2}, 0.1 \mathrm{M} \text { tris, } \\
\text { pH } 7.0\end{array}$ & $\begin{array}{c}30 \% \text { PEG } 4000,0.2 \\
\text { M sodium acetate, } \\
0.1 \text { M tris, pH } 8.3\end{array}$ \\
\hline
\end{tabular}

a Value in brackets indicates high-resolution shell statistics.

${ }^{b} \mathrm{P} / \mathrm{L} / \mathrm{O}$ indicates protein, ligands and others.

${ }^{c}$ rmsd indicates root-mean-square deviation. 
Supplementary method. Synthesis of GS-441524 monophosphate<smiles>C[C@]1(c2ccc3c(N)ncnn23)O[C@H](CO)[C@@H](O)[C@H]1O</smiles><smiles>C[C@]1(c2ccc3c(N)ncnn23)O[C@H](COP(=O)(O)O)[C@@H](O)[C@H]1O</smiles>

\section{GS-441524 monophosphate}

A solution of GS-441524 (43.7 mg, $0.15 \mathrm{mmol})$ in trimethyl phosphate $(1.5 \mathrm{~mL})$ was stirred in a sealed tube under $\mathrm{Ar}$ at $\mathrm{rt}$ for $15 \mathrm{~min}$. The solution was then cooled to $0^{\circ} \mathrm{C}$ and freshly distilled phosphorous oxychloride $(21.2 \mu \mathrm{L}, 0.225 \mathrm{mmol})$ was added dropwise. The resulting solution was stirred at $\mathrm{rt}$ for $1 \mathrm{~h}$. Further $100 \mu \mathrm{L}$ of phosphorous oxychloride were added at $\mathrm{rt}$ and the resulting solution was stirred at rt for $1 \mathrm{~h}$ (full conversion by HPLC). The reaction mixture was quenched with water at $0^{\circ} \mathrm{C}$ and directly purified by preparative HPLC to obtain $43.6 \mathrm{mg}(78 \%)$ of the expected product as a white solid. ${ }^{1 \mathrm{H}}$ $\operatorname{NMR}\left(300 \mathrm{MHz}, \mathrm{D}_{2} \mathrm{O}\right) \delta 7.98(\mathrm{~s}, 1 \mathrm{H}), 7.27(\mathrm{~d}, J=4.9 \mathrm{~Hz}, 1 \mathrm{H}), 7.05(\mathrm{~d}, J=4.9 \mathrm{~Hz}, 1 \mathrm{H}), 4.83(\mathrm{~d}, J=5.2 \mathrm{~Hz}$, $1 \mathrm{H})$, 4.43-4.39 $(\mathrm{m}, 1 \mathrm{H}), 4.31(\mathrm{t}, J=4.7 \mathrm{~Hz}, 1 \mathrm{H}), 4.04-3.92(\mathrm{~m}, 2 \mathrm{H})$; $\mathrm{R}_{\mathrm{f}} \mathrm{HPLC}: 3.4 \mathrm{Min}$ (13 Min from 10 to 95\% MeCN in water (0.1\% formic acid), then $7 \mathrm{~min} 95 \% \mathrm{MeCN}$ ). $95.7 \%$ purity; HRMS (MALDI): $\mathrm{m} / \mathrm{z}$ found. $372.0705[\mathrm{M}+\mathrm{H}]^{+}$(cal. $\mathrm{C}_{12} \mathrm{H}_{15} \mathrm{~N}_{5} \mathrm{O}_{7} \mathrm{P}$ 372.0704).

To record NMR-spectra, the compound was dissolved in $\mathrm{D}_{2} \mathrm{O}$ and measured on Avance 300 from Bruker Corporation (Massachusetts, USA). All chemical shift values are reported in ppm, the multiplicity of the signals assigned as follows: $s$ (singlet), $d$ (duplet), $t$ (triplet) and $m$ (multiplet). Mass spectrometry analysis was performed in positive ion mode by electrospray-ionization (ESI) on a LCMS2020 single quadrupole MS from Shimadzu (Duisburg, Deutschland). Precision mass was measured using MALDI Orbitrap XL from Life Technologies GmbH (Darmstadt, Germany). For purity estimation of the synthesized compounds, a reverse phase high-performance liquid chromatography (RP-HPLC) was performed using the Luna $10 \mu \mathrm{m} \mathrm{C18(2)} 100 \AA$, LC Column $250 \times 4.6 \mathrm{~mm}$ from Phenomenex LTD (Aschaffenburg, Germany) and the analysis was conducted using the Shimadzu prominence module from Shimadzu. Acetonitrile and aqueous formic acid $0.1 \%$ were used as eluents. The established method for purity determination was initiated with $90 \%$ water $(0.1 \%$ formic acid), then a linear gradient from $90 \%$ to $5 \%$ water ( $0.1 \%$ formic acid) for 13 min was chosen, finally additional $7 \mathrm{~min} 5 \%$ water ( $0.1 \%$ formic acid). The flow rate was adjusted to $1.0 \mathrm{~mL} / \mathrm{min}$ and the UV-vis detection occurred at $254 \mathrm{~nm}$ and $280 \mathrm{~nm}$, respectively. 